\title{
Meta-Analysis of Scalp Acupuncture for Acute Hypertensive Intracerebral Hemorrhage
}

\author{
Guo-qing Zheng, MD, PhD, ${ }^{1,2}$ Zhi-Ming Zhao, $\mathrm{PhD}^{3}$, Yan Wang, MD, PhD, ${ }^{2}$ Yong Gu, MD, PhD, \\ Yue Li, PhD, Xing-miao Chen, PhD, Shu-Ping Fu, MD, PhD, and Jiangang Shen, MD, PhD ${ }^{1}$
}

\begin{abstract}
Objectives: Scalp acupuncture (SA) is a commonly used therapeutic approach for primary intracerebral hemorrhage (ICH) in Traditional Chinese Medicine (TCM), but the efficacy and safety of SA therapy are still undetermined. The aim of this study is to systematically evaluate the efficacy and safety of SA therapy for the treatment of acute hypertensive ICH.

Methods: Literature reports with randomized controlled clinical trials and controlled clinical trials on SA therapy for acute hypertensive ICH were searched, and the efficacy and safety of SA therapy were evaluated by using the Cochrane systematic review methods. The primary outcome measures were death or dependency at the end of long-term follow-up (at least 3 months) and adverse events. The secondary outcome measure was neurological deficit improvement at the end of the treatment course.

Results: Seven (7) independent trials (230 patients) were included in this study. All trials described the methods of randomization in which four trials had adequate concealment of randomization at the level of grade A, but no trial included sham acupuncture as a control group. None of the trials included "death or dependency" as a primary outcome measure. Four (4) trials contained safety assessments and stated that no adverse event was found, whereas the other three trials did not provide the information about adverse events. By using random effects statistical model, it was found that patients with acute hypertensive ICH who received SA therapy had significantly improved neurological deficit scores $(Z=4.97, p<0.01)$.

Conclusions: Although SA therapy is widely used to treat acute hypertensive ICH in TCM, the efficacy and safety of SA therapy remain to be further determined. No evidence is available on whether SA therapy can be used to treat acute ICH according to the primary outcome measure. However, SA therapy appears to be able to improve neurological deficit in patients with acute hypertensive ICH.
\end{abstract}

\section{Introduction}

$\mathbf{P}$ RIMARY INTRACEREBRAL HEMORRHAGE (ICH), the most devastating form of stroke, accounts for $10 \%-15 \%$ of stroke victims. ICH has more severe neurological deficits and higher mortality rates than ischemic stroke. ICH secondary to pathologic changes initiated by chronic hypertension is responsible for $\sim 75 \%$ of all cases of primary $\mathrm{ICH}^{1,2} \mathrm{ICH}$ has three main pathophysiologic phases: arterial rupture and hematoma formation, hematoma enlargement, and perihematoma edema. Up to $40 \%$ of the hematomas grow in the first hours postrupture. ICH growth is associated with early clinical deterioration. The volume of ICH directly affects clinical outcome, and approximately one third of ICH cases have a growing volume of hematoma at 3-24 hours after onset., Early interventions to reduce ICH volume might be useful for improving clinical outcome. A recent meta-analysis with 10 randomized controlled trials indicates that surgical treatment could reduce the chances of death or disability. Surgical treatment is a likely benefit for those patients with altered consciousness or neurological deterioration. ${ }^{5}$ However, a large randomized clinical trial (RCT) that involved 1033 patients from 83 centers in 27 countries failed to show a benefit of surgery over conservative treatment in acute $\mathrm{ICH}^{6}{ }^{6}$ Apart from surgical invention, conservative treatments with medications that stop bleeding are potential therapeutic strategies

\footnotetext{
${ }^{1}$ School of Chinese Medicine, University of Hong Kong, Hong Kong, China.

${ }^{2}$ Center of Neurology and Rehabilitation, the Second Affiliated Hospital of Wenzhou Medical College, Wenzhou, China.

${ }^{3}$ Guangdong Provincial Institute of Traditional Chinese Medicine, Guangzhou, China.

Guo-qing Zheng, Zhi-Ming Zhao, and Yan Wang contributed equally to this work.
} 
to prevent or decrease hematoma growth. For instances, factor VII, aminocaproic acid, tranexamic acid, and aprotinin may enhance hemostasis in patients with normal coagulation. ${ }^{7}$ Two (2) RCTs indicate that recombinant factor VIIa is promising to limit hematoma growth and improve outcome, but it was associated with an increase in thromboembolic complications. ${ }^{8,9}$ Until now, there has been no effective therapeutic approach with sufficient evidence to improve the clinical outcome of ICH.

China has a relatively higher incidence of hemorrhagic stroke than Western countries do. ${ }^{10}$ Traditional Chinese Medicine (TCM) was a major available health care system before modern Western medicine was introduced to China. ${ }^{11}$ TCM therapies including acupuncture, herbal medicine, and other nonmedication therapies have been used for treating patients with stroke for thousand years. Scalp acupuncture (SA), also called cranial acupuncture or cephalic acupuncture, is a modality to stimulate different scalp zones corresponding to various brain areas with needles so as to excite the reflex-related nervous tissues and promote recovery of neurological functions. ${ }^{12}$ The SA is one of the major approaches for poststroke disability in acupuncture treatment. The SA was originally developed from cranial acupoints of acupuncture therapy in ancient China, which could be dated back to 5 вс. ${ }^{13}$ Modern SA therapy was derived from traditional acupuncture in combination with the basic principles of neuroanatomy and neurophysiology. Since the World Health Organization (WHO) approved the international standard scalp acupuncture point lines and the brain functional domain projection in $1991,{ }^{14}$ SA therapy has been extensively applied for the treatment of brain disorders in China. ${ }^{15}$ SA therapy is considered an essential therapeutic protocol for rehabilitation of neurological disability in ischemic stroke treatment. ${ }^{16} \mathrm{SA}$ therapy is also used for acute $\mathrm{ICH}$. The first clinical observation on efficacy of SA therapy for acute ICH was described in the 1990s, ${ }^{17,18}$ and now SA therapy has become a popular therapeutic protocol for acute ICH treatment in TCM. However, both experimental studies and clinical trials are not sufficient for evaluating the efficacy and safety of SA therapy for acute ICH treatment.

In this study, to evaluate the efficacy and safety of SA therapy for acute $\mathrm{ICH}$, a literature search was conducted and the existing clinical evidence was reviewed on SA therapy for acute hypertensive ICH by using the Cochrane systematic review methods. This study indicates that SA therapy is a potentially promising therapy for recovery of neurological deficits induced by acute hypertensive $\mathrm{ICH}$, but it will be necessary to conduct well-designed clinical trials to evaluate clinical outcomes in the future.

\section{Study Criteria}

\section{Types of studies}

Randomized controlled clinical trials for evaluating the effects of SA therapy on acute ICH with at least one control group receiving modern standardized treatment or conventional treatment were included in the study.

\section{Types of participants}

Trials that included patients of any age or sex with acute hypertensive ICH (within 14 days since onset) were eligible.
The diagnostic criteria were adopted in accordance with the following: (1) Diagnosis of hypertension was made on the basis of the "Definition and Classification of Hypertension" in 1999 from WHO-International Society of Hypertension (ISH) Guidelines for the Management of Hypertension ${ }^{19}$; (2) Diagnosis of $\mathrm{ICH}$ was made on the basis of the Chinese national criteria of cerebral hemorrhage in Diagnostic Essentials of Various Cerebrovascular Diseases revised at the Fourth National Conference of P. R. China Society of Medicine on Cerebrovascular Diseases in $1995 .^{20}$ As computed tomography/magnetic resonance imaging (CT/MRI) scans are essential for the diagnosis of $\mathrm{ICH}$, all of the patients were examined with CT/MRI scanning in the trials.

\section{Types of interventions}

The clinical trials were included regardless of the number of times of treatment or the length of treatment period, but acupoint selection must be limited to the head, in which ear acupoints should be excluded. Either traditional acupuncture or electroacupuncture was included, regardless of the sources or methods of stimulation (e.g., scalp penetration acupuncture or electrical stimulation). The patients of the control group were given modern conventional treatments. The patients at the trial groups were given SA therapy in addition to the baseline medication similar to the control groups.

\section{Types of outcome measurements}

The primary outcome measurement was death or dependency at the end of long-term follow-up of at least 3 months and adverse events. The secondary outcome measurement was the scores of neurological deficit improvement at the end of the treatment course. The criteria of neurological deficit score were adopted based on the Modified EdinburghScandinavian Stroke Scale, a nationwide accepted scoring system recommended at the Second and revised at the Fourth National Cerebrovascular Diseases Conference in China, including consciousness, gaze, facial paresis, language, walking ability, motor function of arms, legs, and hands. ${ }^{20,21}$ The assessment was conducted in accordance with the reduction in the scores of basic nervous functional deficits and disability degree as follows: Recovery scores-The functional deficit scores were decreased up to $91 \%-100 \%$, and disability degree was at grade 0; Remarkable Improvement-The scores of functional deficit were decreased at $46 \%-90 \%$, and disability degree was at the grade 1-3; Improvement-The scores of functional deficit were decreased at $18 \%-45 \%$; No changeThe scores of functional deficit were decreased or increased at about 17\%; Deterioration-The scores of functional deficit were increased over $18 \%$.

\section{Literature Search}

Computer-based retrieval was performed by using PubMed and Cochrane library (until October 2009) in English, and using full-text database of Chinese periodicals in China National Knowledge Infrastructure (until October 2009) in Chinese by 2 reviewers (Z.M.Z. and G.Q.Z.). The key words used for the search were scalp acupuncture/head acupuncture/cranial acupuncture/cephalic acupuncture/ scalp electric acupuncture/scalp penetration acupuncture and intracerebral hemorrhage. The types of publication 
searched were clinical trials and reviews. The reference lists of all relevant articles were searched for further studies.

\section{Data Extraction and Quality Assessment}

Two (2) review authors (Z.M.Z. and G.Q.Z.) independently assessed all studies. The same 2 review authors independently extracted eligible data from the trial reports onto a data extraction form, and data were cross-checked for accuracy before use. Disagreement was resolved by discussion with a third-party reviewer (J.G.S.) if necessary. The authors of the trials were contacted and asked to provide missing data.

Quality assessment was conducted to determine whether the trials met the following internal validity criteria: (1) method of randomization (truly or quasirandomized); (2) adequate allocation concealment; (3) blinding (both of participants and outcome assessors); (4) participant dropout and loss to follow-up; (5) intention-to-treat (ITT) analysis.

\section{Data Analysis}

Heterogeneity between trial results was tested using a standard $\chi^{2}$ test. The results were reported as Peto-odds ratios with corresponding 95\% confidence intervals for dichotomous data. If continuous data were available, weighted mean difference or standardized mean difference was to be calculated. The figures were obtained from the statistical software provided by the Cochrane Collaboration (RevMan 4.2.10).

\section{Results}

\section{Search strategy and general information}

On the basis of search strategy, we retrieved 21 relevant reports published from 1991 to 2009. After reading through all the reports, we found seven reports of definite or possible RCTs and controlled clinical trials. ${ }^{22-24^{*}, \dagger, t,}$ The remaining 14 reports were excluded because they were uncontrolled observational studies, or duplicate publications, or with patients who did not meet the criteria of the types of participants. A total of 230 patients with acute hypertensive $\mathrm{ICH}$ from seven reports were included. All trials were conducted in China and published from 2003 to 2007 (Table 1).

The ages of patients in the studies were all above 45 years. Each trial included more men than women. All selected trials applied conventional diagnostic criteria for ICH with Chinese National Criteria and for Hypertension with WHO-ISH

*Zhao GJ. Clinical study on acute hypertensive intracerebral hemorrhage with penetrating of scalp-point the study on therapeutic effect and somatosensory evoked potential. MPhil thesis, Heilongjiang University of Traditional Chinese Medicine, 2003.

Zhang SY. Acute hypertensive intracerebral hemorrhage clinical paired observation and serum NSE correlativity study with scalpacupoint penetrating acupuncture therapy. MPhil thesis, Heilongjiang University of Traditional Chinese Medicine. 2005.

Fuan P. Clinical and basic research of security on acute cerebral hemorrhage by acupuncture scalp point-through-point therapy. $\mathrm{PhD}$ thesis, Heilongjiang University of Traditional Chinese Medicine, 2007.

${ }^{\S} \mathrm{Li}$ JJ. Study of scalp penetration acupuncture on serum S100B protein and IGF-1 in blood of patients with acute intracerebral hemorrhage. MPhil Thesis, Heilongjiang University of Traditional Chinese Medicine, 2005.
Guidelines, ${ }^{19,20}$ although many trials also included TCM diagnostic criteria. All patients had undergone a CT/MRI scanning to confirm the diagnosis. The starting time point for SA therapy was within 14 days after stroke. The total period for SA therapy varied from 10 to 28 days. The patients in the included trials received SA therapy plus modern conventional treatment. One (1) trial was performed with surgical intervention. ${ }^{24}$ In all of the trials, neurological deficit was measured with the Modified Edinburgh-Scandinavian Stroke Scale. ${ }^{19,20}$

\section{Quality of the Trials}

All of the trials described the methods of randomization, including random number generator (calculator) and drawing method. Four (4) trials described grade A level of adequate concealment of randomization, in which the patients were allocated according to calculator random numbers sealed in opaque envelopes (see footnotes $*, \uparrow, \uparrow, \S)$. The randomized, single blind, baseline treatment controlled trials were described in these four trials. However, no trial included sham acupuncture as a control group. The remaining three trials did not mention blinding or ITT analysis. ${ }^{22-24}$ All trials conducted a comparable analysis between scalp treatment group and control group, although the raw statistical data are not elaborated in detail.

\section{Death or Dependency}

None of the trials used these as primary outcome measures.

\section{Adverse Events}

No adverse event was mentioned in three trials. ${ }^{22,24(\text { footnote } \dagger)}$ Safety assessments were conducted in the other four trials, and no obvious adverse event was found. ${ }^{21,23(\text { footnotes } *,+, \$)}$ No significant change was observed in the clinical biochemical tests including routine blood, urine and stool tests, myocardial enzymes, liver functions, and renal function be-

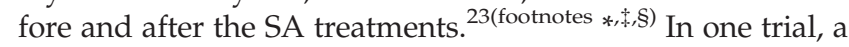
series of studies were conducted on the safety of applying SA therapy for $\mathrm{ICH}$ at acute stage (footnote $\$$ ). There were no significant changes in heart rate, respiration, blood pressure, or oxygen saturation among the selected time points including 2 hours prior to the SA treatments, during the treatments, and 2 hours after the treatments. Interestingly, head CT scan showed that the absorption rate of hematoma in the SA group (76.67\%) was significantly improved compared with those in the control group $(56.67 \%)$ at the time points before and after the treatments. In the SA groups, the abnormal rates of electrocardiography decreased from $73.33 \%$ to $16.67 \%$ after receiving the designed treatments, whereas in the control group it was decreased from $70 \%$ to $43.33 \%$ after the designed treatments.

\section{Neurological Deficit Improvement}

The seven independent trials $22-24$ (footnotes $*, \dagger, \$)$ did not show homogeneity in the consistency of the trial results $\left(\chi^{2}=17.43, p<0.01\right)$. Thus, the random-effect model should be used for statistical analysis. In the random-effect statistical model, the combined effects of seven independent trial results showed that patients with acute hypertensive $\mathrm{ICH}$ 


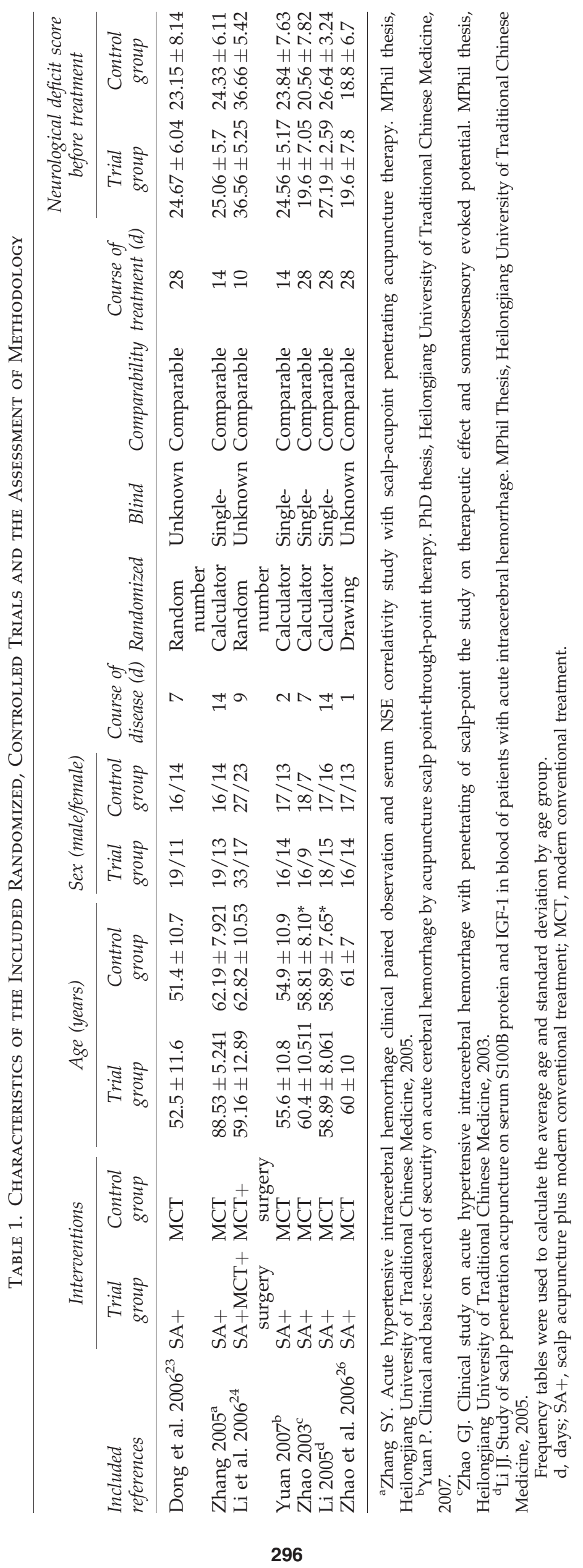




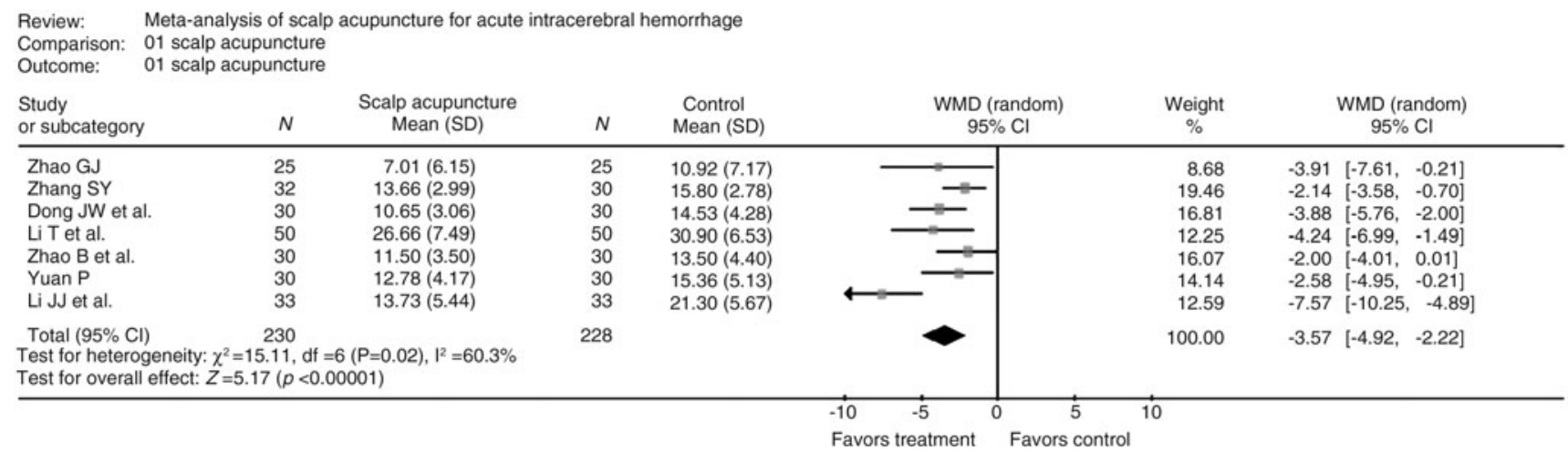

FIG. 1. Neurologic deficit improvement after treatment with scalp acupuncture versus control. SD, standard deviation; WMD, weighted mean difference; CI, confidence interval.

receiving SA therapy had significantly improved neurological deficit scores when compared with the control group $(Z=4.97, p<0.01$, Fig. 1).

\section{Bias Determination}

Publication bias was found in the included seven independent references. One (1) trial had serious bias, whereas the other six trials had relatively slight bias in the evaluations of the clinical outcomes. These six trials also confirmed the positive effects of SA therapy for improving neurological deficit scores in acute hypertensive ICH (Fig. 2).

\section{Discussion}

This is the first meta-analysis for evaluating the clinical outcome of SA therapy for acute ICH. The results indicate that SA therapy appears to be effective for improving neurological deficit scores in patients with acute hypertensive ICH.

Experimental and clinical studies suggest that SA therapy might be a useful therapeutic approach for poststroke neurological dysfunctions, but its mechanisms still are largely unknown. Theoretically, SA therapy can stimulate specific scalp areas corresponding to the functional areas of cerebral cortex. SA needle stimulation can effectively raise blood flow volume in the common carotid artery, leading to an increase of the energy supply of the cerebral blood circulation in patients who have had a stroke. ${ }^{25}$ Functional magnetic resonance imaging (fMRI) study revealed that SA therapy in- creased brain oxygen content in healthy volunteers. ${ }^{26}$ Experimental stroke studies suggest that SA stimulation could regulate the expressions of cytokines, inflammation factors, and transcriptional factors in ischemia-reperfused brains. For instance, in rat models of acute cerebral ischemiareperfusion injury, SA stimulation suppressed the expressions of nuclear factor $-\kappa \mathrm{B}$, cyclo-oxygenase- 2 , tumor necrosis factor- $\alpha$, and interleukin- $1 \beta$, inhibited leukocyte infiltration, and enhanced the expression of transforming growth factor- $\beta 1$ in rat brains, subsequently attenuating cerebral ischemia-reperfusion injury and improving neurofunctional rehabilitation. ${ }^{27,28}$ A fMRI study on a hypertensive stroke rat model revealed that SA stimulation reduced vasogenic edema and reduced neurological dysfunction. ${ }^{29}$ As most studies focus on ischemic stroke in current reports, studies on SA therapy for acute ICH are insufficient experimentally and clinically. The effectiveness and safety of SA therapy for acute ICH treatment have not been evaluated with current rigorous international standards. Therefore, it is very urgent and important to assess the quantity, quality, and overall strength of SA therapy for acute ICH treatment.

In this meta-analysis, we found that the primary outcomes were inadequately designed in all of the included clinical trials. There were unknown dropout rates and definitional vagueness in the measurement. The duration of therapy and the follow-up period were not long enough to achieve conclusive results. None of the studies included endpoints such

Review: Meta-analysis of scalp acupuncture for acute intracerebral hemorrhage

Comparison: 01 scalp acupuncture

Outcome: 01 scalp acupuncture

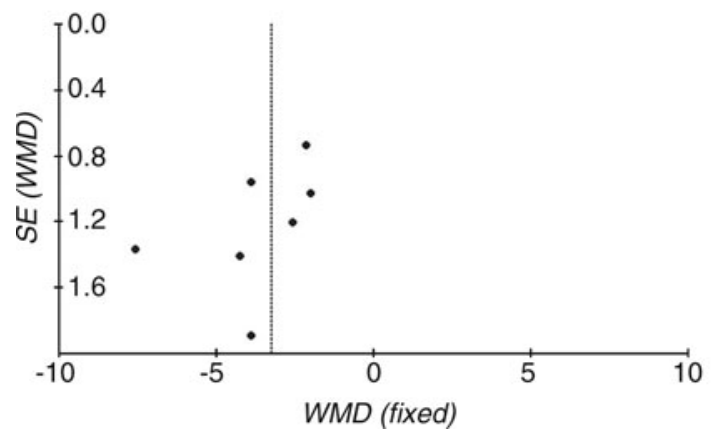

FIG. 2. Funnel plot of the comparison of clinical effects of scalp acupuncture with those of control groups. SE, standard error; WMD, weighted mean difference. 
as the rates of death, the rate of dependency, survival times, or reduction in length of staying in hospital or long-term care. Thus, there was no evidence available about the effect of SA therapy on the rates of death or dependency at the end of follow-up (at least 3 months). The other primary outcome was adverse effects. Owing to the rapid onset, the dangerous condition and high mortality of hemorrhagic stroke, some academics were skeptical about SA for acute ICH (see footnote $\$$ ). They remarked that SA was not suitable for acute ICH. The major concern was that external stimulus might affect the stability of hematoma and increase the risks of cerebral edema and hematoma growth. According to the safety assessment of the four trials in this systematic review, $\left.{ }^{23(f o o t n o t e s} * \pm, \$\right)$ SA therapy for acute $\mathrm{ICH}$ is generally safe. However, intensive investigations with relatively large scales and high standard in experimental design are prerequired before SA therapy is recommended for common clinical application.

The most noticeable finding is that SA therapy was similarly effective in all of the clinical trials based on the secondary outcomes in the improvement of neurological deficit scores. The effects could be real, but they could also be attributable to the potential bias and to the methodological quality of the included trials. Publication bias could be a factor. There are several methodological limitations in the clinical trials. First, the blinding procedures could be a factor because all of the trials did not design sham acupuncture as the control group. In the design of clinical trials for acupuncture research, the comparison of SA and sham treatments with the blind or double-blind clinical experiments is extremely difficult. With a restrict standard of blind control in RCTs, if the patients have a sensation at the points of needle contact, it could not be considered a valid blind control. The same problem arises in double-blinding procedures used in physical therapy, surgical procedures, dentistry, and so on. Therefore, developing an effective method for sham acupuncture will be critical in the future. Second, the included trials were generally of small sample size. None of the trials reported the method of determination of the sample size. Third, the scheduled treatment period ranged from 14 days to 28 days in the included trials. All of the follow-ups were terminated at the end of the treatment period. Three (3) months after the onset of stroke could be an appropriate time to measure the neurological outcome, because the recovery of neurological deficit occurred quickly in first 3 months after symptom onset. Nevertheless, although current clinical trials could not lead to drawing a conclusion, the results do provide some promising data indicating that SA therapy can improve outcomes in the neurological deficit scores. Given that SA therapy is widely used for acute $\mathrm{ICH}$ by TCM practitioners, a blind placebo-controlled RCT including primary and second outcomes becomes a timely important issue. In addition, using fMRI or other brain imaging modalities to monitor hematoma growth in experimental ICH animal models and clinical trials is also a critical research topic in this field.

\section{Conclusions}

In conclusion, insufficient evidence is available to support the routine use of SA for acute ICH according to the primary measure of efficacy. However, SA therapy appears to be generally safe and can be used in the acute stage of ICH. SA therapy may have beneficial effects on neurological impairment, but this efficacy needs to be further confirmed by methodologically rigorous trials. The key for evidence-based practices is to design and conduct appropriate RCTs with adequate concealment of allocation, blinding of outcome assessors and sham acupuncture control, and establishment of a consensus on standardized relevant outcome measurements. The primary outcome and relatively long-term follow-up are also important. If SA therapy can improve the clinical outcome of acute ICH as confirmed with the evidence-based medicine approaches, SA therapy will be an important contribution of TCM to human health care worldwide.

\section{Acknowledgments}

This work is supported by a donation from the family of Dr. Wong B.L. donation (S.J.G.) and the Key Project Grant of Wenzhou Municipal Science and Technology Bureau in Zhejiang Province of China (Y20070038, ZGQ).

\section{Disclosure Statement}

No competing financial interests exist.

\section{References}

1. Brott $\mathrm{T}$, Thalinger $\mathrm{K}$, Hertzberg V. Hypertension as a risk factor for spontaneous intracerebral hemorrhage. Stroke 1986;17:1078-1083.

2. McCormick WF, Rosenfield DB. Massive brain hemorrhage: A review of 144 cases and an examination of their causes. Stroke 1973;4:946-954.

3. Brott T, Broderick J, Kothari R, et al. Early hemorrhage growth in patients with intracerebral hemorrhage. Stroke 1997;28:1-5.

4. Davis SM, Broderick J, Hennerici M, et al. Hematoma growth is a determinant of mortality and poor outcome after intracerebral hemorrhage. Neurology 2006;66:1175-1181.

5. Prasad K, Mendelow AD, Gregson B. Surgery for primary supratentorial intracerebral hematoma: A meta-analysis of 10 randomized controlled trials. Stroke 2009;40:e624-e626.

6. Mendelow AD, Gregson BA, Fernandes HM, et al.; STICH investigators. Early surgery versus initial conservative treatment in patients with spontaneous supratentorial intracerebral haematomas in the International Surgical Trial in Intracerebral Haemorrhage (STICH): A randomised trial. Lancet 2005;365:387-397.

7. Ferro JM. Update on intracerebral haemorrhage. J Neurol 2006;253:985-999.

8. Mayer SA, Brun NC, Begtrup K, et al. for the Recombinant Activated Factor VII Intracerebral Hemorrhage Trial Investigators. Recombinant activated factor VII for acute intracerebral hemorrhage. NEJM 2005;352:777-785.

9. Mayer SA, Brun NC, Broderick J, et al. for the Europe/ Austral Asia NovoSeven ICH Trial Investigators. Safety and feasibility of recombinant factor VIIa for acute intracerebral hemorrhage. Stroke 2005;36:74-79.

10. Jiang B, Wang WZ, Chen $\mathrm{H}$, et al. Incidence and trends of stroke and its subtypes in China: Results from three large cities. Stroke 2006;37:63-68.

11. World Health Organization Western Pacific Region, Manila, Philippines. WHO International Standard Terminologies on Traditional Medicine in the Western Pacific Region. Geneva: World Health Organization, 2007. 
12. Zheng GQ. Methodological standards for experimental research on stroke using scalp acupuncture. Acupunct Electrother Res 2009;34:1-13.

13. Jin S. Scalp Acupuncture Wall Chart [in English and Chinese]. Beijing: People's Medical Publishing House, 2006.

14. WHO Scientific Group on International Acupuncture Nomenclature; World Health Organization. A Proposed Standard International Acupuncture Nomenclature: Report of a WHO Scientific Group. Geneva: World Health Organization, 1991.

15. Chen XJ. Progress in clinical application of scalp-acupuncture. World J Acup Mox 2001;11:3-6.

16. Li FL, Lu FY. Recent ten years research progress in scalpacupuncture therapy for stroke treatment. Chin J Rehabil Theory Practice 2005;11:451-452.

17. Dong G, Guan L, Sun F, Wang J. Comparative study on therapeutic effect of scalp-acupuncture in treating acute cerebral hemorrhage. Int J Clin Acupunct 1992;3:31-38.

18. Dong GR, Guan LB, Liu M. Observation of 48 cases of cerebral hemorrhage patients with scalp acupuncture therapy. Zhongguo Zhen Jiu 1990;10:19-20.

19. World Health Organization-International Society of Hypertension Guidelines for the Management of Hypertension. Guidelines Subcommittee. J Hypertens 1999;17:151-183.

20. Chen QT. Classification, diagnostic criteria and evaluation of neurological impairment for stroke patients. Chin J Neurol 1996;29:376-338.

21. Wu B, Liu M, Liu H, et al. Meta-analysis of traditional Chinese patent medicine for ischemic stroke. Stroke 2007;38:1973-1979.

22. Zhao B, Liu RH, Wang ZL. Effects of electro-acupuncture on the changes of serum neuron-specific enolase and nerve function of hypertensive intracerebral hemorrhage patients. Chin J Clin Rehabil 2006;10:7-9.

23. Dong JW, Bao CL, Gong WZ. Clinical research of efficacy on acute cerebral hemorrhage by acupuncture scalp pointthrough-point therapy. Clin J Tradit Chin Med 2006;18:341-342.
24. Li T, Zhao JG, Tian GJ, et al. Clinical observation on effect of acupuncture on nervous functions of the patient after operation of hypertensive cerebral hemorrhage. Zhongguo Zhen Jiu 2006;26:247-249.

25. Li L, Gong JQ, Ding GH, et al. Effect of multiple paralleled acupuncture needles stimulation of scalp points on hemodynamics and blood flow energy of the common carotid artery in stroke patients. Zhen Ci Yan Jiu 2009;34:334-338.

26. Park SU, Shin AS, Jahng GH, et al. Effects of scalp acupuncture versus upper and lower limb acupuncture on signal activation of blood oxygen level dependent (BOLD) fMRI of the brain and somatosensory cortex. J Altern Complement Med 2009;15:1193-1200.

27. Zhou L, Zhang HX, Wang Q, et al. Effect of scalp acupuncture on the expression of NF-kappaB mRNA, COX-2 mRNA and their proteins in rats with acute cerebral ischemia-reperfusion injury. Zhen Ci Yan Jiu 2009;34:304-308.

28. Zhang HX, Liu LG, Zhou L, et al. Effect of scalp acupuncture on inflammatory response in rats with acute cerebral ischemia-reperfusion injury. Zhong Xi Yi Jie He Xue Bao 2007; 5:686-691.

29. Inoue I, Fukunaga M, Koga K, et al. Scalp acupuncture effects of stroke studied with magnetic resonance imaging: Different actions in the two stroke model rats. Acupunct Med 2009;27:155-162.

Address correspondence to: Jiangang Shen, MD, PhD School of Chinese Medicine University of Hong Kong 10 Sassoon Road Hong Kong China

E-mail: shenjg@hkucc.hku.hk 
